\title{
Objective evaluation of ERCP procedures: a simple grading scale for evaluating technical difficulty
}

\author{
K Ragunath, L A Thomas, W Y Cheung, P D Duane, D G Richards
}

Postgrad Med J 2003;79:467-470

\begin{abstract}
Background and objective: Endoscopic retrograde cholangiopancreatography (ERCP) is a technically demanding endoscopic procedure that varies from a simple diagnostic to a highly complex therapeutic procedure. Simple outcome measures such as success and complication rates do not reflect the competence of the operator or endoscopy unit, as case mix is not taken into account. A grading scale to assess the technical difficulty of ERCP can improve the objectivity of outcome data.

Methods: A I to IV technical difficulty grading scale was constructed and applied prospectively to all ERCPs over a 12 month period at a single centre. The procedures were performed by two senior trainees and two experienced consultants (trainers). The grading scale was validated for construct validity and inter-rater reliability at the end of the study using the $\chi^{2}$ test and $\kappa$ statistics.

Results: There were 305 ERCPs in 259 patients over the 12 months study period (males: 112, females: 147 , age range 17-97, mean 70.3 years). There was overall success in $244(80 \%)$ procedures with complications in $13(4 \%)$ : bleeding in five $(1.6 \%)$, cholangitis in one $(0.3 \%)$, pancreatitis in five $(1.6 \%)$ and perforation in two $(0.7 \%)$. Success rate was highest for grade I, $49 / 55(89 \%)$, compared with grade IV procedures, $8 / 11$ (73\%). There was a significant linear trend towards a lower success rate from grade I to IV $(p=0.021)$ for trainees, but not for trainers. Complications were low in grade I, II, and III procedures, 12/295(4\%), compared with grade IV procedures, $1 / 11(9 \%)$. The inter-rater reliability for the grading scale was good with a substantial agreement between the raters $(\kappa=0.68$, $\mathrm{p}<0.001$ ).

Conclusion: Success and complications of ERCP by trainees are influenced by the technical difficulty of the procedure. Outcome data incorporating a grading scale can give accurate information when auditing the qualitative outcomes. This can provide a platform for structured objective evaluation.
\end{abstract}

See end of article for authors' affiliations

Correspondence and reprint requests to: Dr Krish Ragunath, Division of Gastroenterology, Floor C South Block, Queens Medical Centre, University Hospital, Nottingham NG7 2UH, UK:

k.ragunath@nottingham.ac.uk

Submitted

22 November 2002

Accepted 30 April 2003

$E^{n}$ ndoscopic retrograde cholangiopancreatography (ERCP) is an advanced endoscopic procedure that is not only technically challenging, but also associated with a risk of serious complications. The procedure itself varies from a simple, straightforward diagnostic ERCP to a highly specialised therapeutic ERCP. A recent survey in the UK showed wide differences between endoscopists in success rates (from $76 \%$ to $95 \%$ ) and serious complications ( $0 \%$ to $16 \%){ }^{.}$It is far from acceptable to have such a variation in practice and outcomes in any health care system. Studies have shown that skill and experience have a huge impact on the outcomes of ERCP. ${ }^{2}$ Success and complications depend not only on the endoscopist's experience, but also on the technical difficulty. Auditing ERCP procedures by simple outcome measures such as complication and success, without taking into account the experience and technical difficulty, does not reflect the actual competence of the unit or an individual endoscopist. Units dealing with advanced, therapeutic ERCP procedures may not be achieving the same success rate as units dealing with simple, straightforward ERCPs. There is a pressing need for objective evaluation of medical interventions. It is essential that we have yardsticks to measure the outcomes in a more objective manner. In a consensus conference, ERCP complication definitions have been standardised by Cotton et al. ${ }^{4}$ In addition, Fleischer et al have developed a system for classifying and grading such complications by quantifying their negative repercussions. ${ }^{5}$ Similarly Jowell et al have developed a system to assess technical competence and experience in ERCP procedures. ${ }^{6}$ A grading scale to assess the technical difficulty would complement this to achieve an overall objective evaluation. This will allow centre-to-centre and endoscopist-toendoscopist comparisons. Our aim was to introduce and validate a technical difficulty grading scale for ERCP procedures that can be used as a simple tool to objectively evaluate the procedures.

\section{METHOD}

Demographic and procedural data relating to all ERCP procedures performed in Morriston Hospital over a 12 month period from September 1999 to August 2000 were collected prospectively. All the procedures were performed by two experienced consultants (trainers) with experience of over 1000 ERCPs and two senior trainees with experience of 100-125 ERCPs. Whenever the trainee failed, a trainer took over and completed the procedure and the final outcome was recorded. Trainees were allowed a maximum of 15 minutes; where there was no adequate progress in the procedure it was deemed a failure by the trainee. Only when the entire intended procedure (for example, sphincterotomy, stent insertion) was completed without the trainer's assistance was the procedure classed as trainee success. Overall success was defined as completion of the intended procedure (for example, sphincterotomy, stent insertion) and not just cannulating the desired duct. Technical difficulty of the procedure was recorded according to a grading system devised by us (table 1). Complications (within 30 days) were collected from the hospital records as well as from the general practitioner records and were graded according to a grading system introduced by Cotton et al. ${ }^{4}$ All procedural data were collected, using specially designed forms, by the endoscopist at the end of each procedure, and finally entered

Abbreviations: ERCP, endoscopic retrograde cholangiopancreatography; $\mathrm{MRCP}$, magnetic resonance cholangiopancreatography 
Table 1 Morriston Hospital ERCP grading scale

\begin{tabular}{|c|c|}
\hline Procedure & Grade \\
\hline Diagnostic ERCP & 1 \\
\hline $\begin{array}{l}\text { Biliary sphincterotomy (bowstring sphincterotome), balloon sphincteroplasty, removal of } \\
\text { extrahepatic stones } \leqslant 1 \mathrm{~cm} \text { using basket and/or balloon }\end{array}$ & ॥ \\
\hline $\begin{array}{l}\text { Precut sphincterotomy, large stones removal }(>1 \mathrm{~cm}) \text {, intrahepatic stone removal, mechanical } \\
\text { lithotripsy, stricture dilatation, cytology, stent insertion, and nasobiliary drain }\end{array}$ & III \\
\hline $\begin{array}{l}\text { Sphincter of Oddi manometry, diagnostic and therapeutic ERCP after Billroth II surgery, minor } \\
\text { papilla sphincterotomy, endoscopic ampullectomy and all pancreatic duct therapeutic procedures. } \\
\text { Cholangioscopy, laser lithotripsy, electrohydrolic lithotripsy, combined procedures (PTC and } \\
\text { ERCP), and other advanced bile duct therapeutic procedures }\end{array}$ & IV \\
\hline
\end{tabular}

Table 2 Summary of all ERCP procedures according to the grading scale

\begin{tabular}{llll}
\hline Procedure & No (\%) & Success (\%) & Complication (\%) \\
\hline Grade I & $55(18)$ & $49(87)$ & $2(4)$ \\
Grade II & $86(28)$ & $65(75.5)$ & $3(5)$ \\
Grade III & $153(50)$ & $122(80)$ & $7(5)$ \\
Grade IV & $11(4)$ & $8(63)$ & $1(9)$ \\
Total & 305 & $244(80)$ & $13(4)$ \\
\hline
\end{tabular}

into our computer database. The construct validity of the grading scale was assessed by testing the following hypothesis: (a) the greater the technical difficulty of the procedure, the lower the success, (b) the greater the technical difficulty of the procedure, the higher the complication rate. Inter-rater reliability was tested at the end of the study as follows: (i) patient details and endoscopist identification were deleted on all ERCP reports, (ii) reports were initially graded by the endoscopists who performed the procedure and then, were randomly allocated to the endoscopists for regrading without knowledge of the previous grading or the endoscopist. The statistician involved in the study (WYC) did this blinded report review. Statistical analyses were done using SPSS 10 software (SPSS Inc, Chicago, IL, USA). The $\chi^{2}$ test was used to test the hypothesis; $\kappa$ statistics were calculated to measure the inter-rater reliability.

\section{RESULTS}

A total of 305 ERCPs were performed in 259 patients over the 12 month study period. There were 112 males and 147 females, age range $17-97$ years (mean 70.3 years). The overall success was $244(80 \%)$ with complications in $13(4 \%)$ comprising pancreatitis in five (1.6\%), bleeding (mild) in five $(1.6 \%)$, perforation in two $(0.7 \%)$, and cholangitis in one $(0.3 \%)$. All the complications were graded as mild according to the grading system by Cotton et al. ${ }^{4}$ A summary of ERCP procedures in the various grades including the success and complication rates are given in table 2 .

Regarding hypothesis (a) our findings indicate a significant linear trend towards a lower success rate from grade I to IV $(p=0.021)$ for trainees (table 3$)$. Success rate for trainees decreased from $85 \%$ for grade I to $50 \%$ for grade IV, whereas this trend was not seen for trainers where success was equally good for all grades (table 3). For trainees, the difference in success rate between grade II and grade III procedures $(6 \%)$ was smaller than that between grade I and grade II ( $14 \%)$ or grade III and grade IV(15\%) (table 3 ).

The overall complication rate was low (4\%) (table 2). There were insufficient data to test hypothesis (b) directly. However, complications were low in grade I, II, and III procedures, $12 / 295(4 \%)$, compared with grade IV procedures, $1 / 11$ (9\%) (table 2). This was in agreement with the hypothesis that the greater the technical difficulty, the higher the complication rate, though the difference was not statistically significant. The overall 30 day mortality was four deaths out of 259 patients $(1.5 \%)$. Three patients succumbed to their hepatobiliary or pancreatic malignancy and one patient died of respiratory failure secondary to chronic lung disease. There were no procedure related deaths.

The inter-rater reliability was good. Agreement between the two raters for grade I procedures was $93 \%, 86 \%$ for grade II procedures, $68 \%$ for grade III procedures, and $91 \%$ for grade IV procedures (table 4). There was substantial agreement between the raters $(\kappa=0.68, p<0.001)$.

\section{DISCUSSION}

Clinical effectiveness, risk management, outcomes, and audit are now a major influence on any health care provider. Undoubtedly ERCP is one of the most difficult and technically challenging endoscopic procedures. Apart from being diagnostic it can provide vital therapeutic options that can prevent or complement open surgery on the pancreatic and hepatobiliary tract. It is imperative that we have robust measures to objectively assess these procedures. The focus of attention in this study was to introduce a technical difficulty grading scale that can be easily understood and applied in everyday practice to facilitate audit and outcomes research. The success rate in ERCP not only depends on the endoscopist but also on the technical difficulty of the procedure. It is well known that not all endoscopists or endoscopy units undertake difficult therapeutic ERCP. It is not ideal to compare all endoscopists or endoscopy units as one cohort because of this case mix scenario. The success and complication rates can vary. The grading scale we have introduced is an easily understandable I to IV scale to qualitatively assess ERCP procedures, which in turn will give an overall view of the type of work done in an endoscopy unit or by an individual endoscopist.

The grading scale was able to differentiate the simplest (grade I) from the most difficult (grade IV) procedures based on the success rate, although this was not the case for grade II and III procedures. Success for trainees was highest with grade I with a significant linear trend towards lower success for higher grade procedures. However, this was not the case for the trainers. This may be due to the limited number of procedures resulting in a type II error or the scale is truly specific for the trainees. Though not statistically significant, it is interesting to note that complications were low in grade I, II, and III procedures $(4 \%)$ compared with grade IV procedures $(9 \%)$. This again, seems to complement the grading scale. A larger study population should be able to overcome this shortfall.

The inter-rater reliability tested as part of the validation process showed a high degree of agreement between the raters. The discriminatory power between grade II and grade III procedures of our proposed scale was weaker and there was 
Table 3 Summary of ERCP procedures performed by trainers and trainees according to the grading scale

\begin{tabular}{|c|c|c|c|c|}
\hline \multirow[b]{2}{*}{ Procedure } & \multicolumn{2}{|l|}{ Success (\%) } & \multicolumn{2}{|c|}{ Complication (\%) } \\
\hline & Trainer & Trainee & Trainer & Trainee \\
\hline Grade I & $20 / 21(95)$ & $29 / 34(85)$ & $0 / 21(0)$ & $2 / 34(6)$ \\
\hline Grade II & $30 / 37$ (81) & $35 / 49(71)$ & $1 / 37$ (3) & $3 / 49(6)$ \\
\hline Grade III & $74 / 79$ (94) & $48 / 74(65)$ & $2 / 79$ (3) & $4 / 74(5)$ \\
\hline Grade IV & $6 / 7(86)$ & $2 / 4(50)$ & $1 / 7(14)$ & $0 / 4(0)$ \\
\hline
\end{tabular}

\begin{tabular}{lllll} 
Table 4 & Level of agreement between raters & & \\
\hline & Grade I (\%) & Grade II (\%) & Grade III (\%) & Grade IV (\%) \\
\hline Raters agree & $51(93)$ & $74(86)$ & $104(68)$ & $10(91)$ \\
Raters disagree & $4(7)$ & $12(14)$ & $49(32)$ & $1(9)$ \\
Total & 55 & 86 & 153 & 11 \\
\hline
\end{tabular}

a lower level of agreement between raters for grade III procedures, which indicated the need to further examine the differences between grade II and grade III procedures. There was $32 \%$ disagreement for the grade III procedures. The second rater graded the procedures retrospectively and blindly based on the ERCP report, having not been present during the procedure. This could be due to the size of the stones when exact measurements of the stones are not mentioned in the report. We have not attempted to look into this since this was not anticipated and we have not incorporated this in our study protocol. Combining grade II and III procedures should further simplify the grading scale. Results of our tests on construct validity and inter-reliability of the scale showed enough evidence to justify further work to refine the scale.

We have started our grading scale with diagnostic ERCP as grade I, which may become a rarity in the future with the advent of other imaging modalities like magnetic resonance cholangiopancreatography (MRCP) and endoscopic ultrasound. MRCP and endoscopic ultrasound are not widely available, are more expensive, and need extra technical expertise. Also there are limitations-for example, endoscopic ultrasound cannot image the intrahepatic ducts and hilum with clarity when compared with ERCP. ${ }^{78}$ MRCP is not accurate in non-dilated ducts when compared with ERCP and can still miss small stones $<5 \mathrm{~mm}$. MRCP cannot be performed in claustrophobic patients and patients with pacemakers and other metal implants. MRCP and endoscopic ultrasound cannot totally replace ERCP since a select group of patients will still need diagnostic ERCP. Hence we would need a grade to classify diagnostic ERCP.

Factors such as size of papilla, position with respect to diverticulum, and distortion of the duodenum are variables that can be inconsistent. Only periampullary duodenal diverticulum has been shown to increase the failure rate. ${ }^{9}$ The presence or absence of periampullary duodenal diverticulum can be added as a modifier in each grade of this grading scale, although this will increase the complexity of the grading scale.

We are aware that two centres in the United States have introduced technical difficulty grading scales. Schutz and Abbott were the first investigators to introduce a technical difficulty grading scale. ${ }^{10}$ They introduced a five point grading scale (I to V with increasing difficulty) and a " $\mathrm{B}$ " modifier to identify previous failed procedures. They applied this retrospectively and then prospectively on their patient population and showed a decreased success rate in grade V and V B procedures when compared with grade I to IV procedures.
Madhotra et al have introduced a modified degree of difficulty grading scale and applied this to data from seven North American centres using the GI Trac database. ${ }^{11}$ They applied a three tier grading scale to 8000 ERCP procedures and concluded that success rates were lower in higher grade procedures. The grading scale introduced by the above two groups is slightly more complicated than ours, and has not been validated. Our grading scale is another preliminary attempt to grade the technical difficulty in ERCP procedures. It is limited by the number of procedures and can be further simplified by combining the grade II and III procedures. It is also noticeable that our proposed scale is particularly sensitive to the performances of trainees. Trainees could start training in grade I procedures and gradually progress up the scale as competence is gained. It could be a useful instrument to assist training and help training units to identify areas where individual trainee would require further support.

In conclusion, success and complications of ERCP in trainees are influenced by the technical difficulty of the procedure. Outcome data incorporating a grading scale can give accurate information when auditing the qualitative outcomes. We have made significant progress in this area. A consensus conference should be addressed to finalise a validated, internationally acceptable, easily understandable, reproducible and robust grading scale, which can be applied in everyday practice as a tool to objectively analyse ERCP procedures.

\section{ACKNOWLEDGEMENT}

Presented in part at the BSG meeting, March 2001, Glasgow, United Kingdom (Gut 2001;48(suppl 1):A96).

\section{Authors' affiliations}

K Ragunath, L A Thomas, P D Duane, Department of Gastroenterology, Morriston Hospital, Swansea, UK W Y Cheung, School of Postgraduate Studies in Medical and Health Care, University of Wales, Swansea, UK

D G Richards, Department of Radiology, Morriston Hospital, Swansea, UK

\section{REFERENCES}

1 Tanner AR. ERCP: present practice in a single region. Eur J Gastroenterol Hepatol 1996:8:145-8.

2 Loperfeido S, Angelini G, Benedetti G, et al. Major early complications from diagnostic and therapeutic ERCP: a prospective multicenter study. Gastrointest Endosc 1998;48:1-10. 
3 Rabenstein T, Schneider HT, Nicklas M, et al. Impact of skill and experience of the endoscopist on the outcome of endoscopic sphincterotomy techniques. Gastrointest Endosc 1999:50:628-36.

4 Cotton PB, Lehman G, Vennes J, et al. Endoscopic sphincterotomy complications and their management: an attempt at consensus. Gastrointest Endosc 1997;37:383-93.

5 Fleischer DE, Van de Mierop F, Eisen GM, et al. A new system for defining endoscopic complications emphasizing the measure of importance Gastrointest Endosc 1997:45:128-33.

6 Jowell PS, Baillie J, Stanley BM, et al. Quantitative assessment of procedural competence. A prospective study of training in endoscopic retrograde cholangiopancreatography. Ann Intern Med 1996; 125:983-9.
7 Palazzo L, O'Toole D. EUS in common bile duct stones. Gastrointest Endosc 2002:56:S49-57.

8 Baillie J. Magnetic resonance cholangiopancreatography: the

gastroenterologist's perspective. Gastrointest Endosc 2002;55:S13-15.

9 Lobo DN, Balfour TW, Iftikhar SY. Periampullary diverticula:

consequences of failed ERCP. Ann R Coll Surg Engl 1998;80:326-31.

10 Schutz SM, Abbott RM. Grading ERCPs by degreee of difficulty: a new concept to produce more meaningful outcome data. Gastrointest Endosc 2000.51:535-9.

11 Madhotra R, Cotton PB, Vaughn J, et al. Analyzing ERCP practice by a modified degree of difficulty scale: a multicentre database analysis. Am $J$ Gastroenterol 2000;95:2480-1 (abstract).

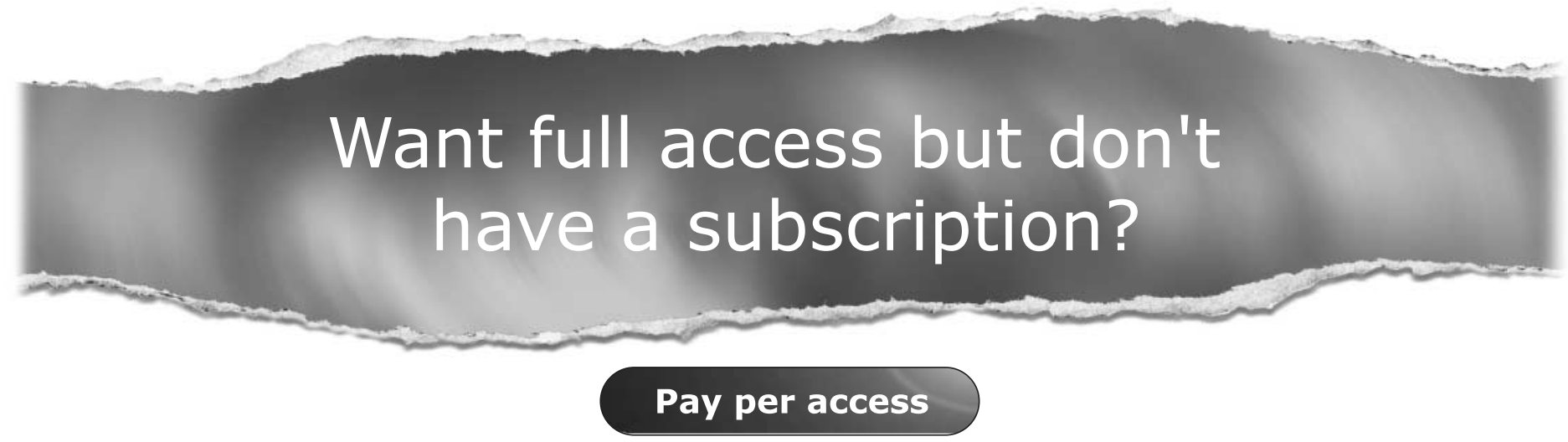

For just US\$25 you can have instant access to the whole website for 30 days. During this time you will be able to access the full text for all issues (including supplements) available. You will also be able to download and print any relevant pdf files for personal use, and take advantage of all the special features Postgraduate Medical Journal online has to offer.

www.postgradmedj.com 